Band Gap Tuning via Lattice Contraction and Octahedral Tilting in Perovskite Materials for Photovoltaics

Peer-reviewed author version

Prasanna, Rohit; Gold-Parker, Aryeh; Leijtens, Tomas; CONINGS, Bert;

BABAYIGIT, Aslihan; BOYEN, Hans-Gerd; Toney, Michael F. \& McGehee, Michael

D. (2017) Band Gap Tuning via Lattice Contraction and Octahedral Tilting in

Perovskite Materials for Photovoltaics. In: JOURNAL OF THE AMERICAN

CHEMICAL SOCIETY,139 (32), p. 11117-11124.

DOI: $10.1021 /$ jacs.7b04981

Handle: http://hdl.handle.net/1942/24071 


\title{
Band Gap Tuning via Lattice Contraction and Octahedral Tilting in Perovskite Materials for Photovoltaics
}

Rohit Prasanna $^{1}$, Aryeh Gold-Parker ${ }^{2,3}$, Tomas Leijtens ${ }^{1 *}$, Bert Conings ${ }^{4}$, Aslihan Babayigit ${ }^{4}$, Hans-Gerd Boyen ${ }^{4}$, Michael F. Toney ${ }^{3}$, and Michael D. McGehee ${ }^{1 *}$

1. Department of Materials Science, Stanford University, 476 Lomita Mall, Stanford CA 94305, United States

2. Department of Chemistry, Stanford University, Stanford CA 94305, United States

3. Stanford Synchrotron Radiation Lightsource, SLAC National Accelerator Laboratory, Menlo Park CA 94025, United States

4. Institute for Materials Research (IMO), Hasselt University, Wetenschapspark 1, 3590 Diepenbeek, Belgium

* Corresponding authors. Email: $\underline{\text { mmcgehee@stanford.edu, toleijtens@ gmail.com }}$

\begin{abstract}
Tin and lead iodide perovskite semiconductors of the composition $\mathrm{AMX}_{3}$, where $\mathrm{M}$ is a metal and $\mathrm{X}$ is a halide, are leading candidates for high efficiency low cost tandem photovoltaics, in part because they have band gaps that can be tuned over a wide range by compositional substitution. We experimentally identify two competing mechanisms through which the A-site cation influences the band gap of 3D metal halide perovskites. Using a smaller A-site cation can distort the perovskite lattice in two distinct ways: by tilting the $\mathrm{MX}_{6}$ octahedra, or by simply contracting the lattice isotropically. The former effect tends to raise the band gap, while the latter tends to decrease it. Lead iodide perovskites show an increase in band gap upon partial substitution of the larger formamidinium with the smaller cesium, due to octahedral tilting. Perovskites based on tin, which is slightly smaller than lead, show the opposite trend - they show no octahedral tilting upon Cs-substitution, but only a contraction of the lattice, leading to progressive reduction of the band gap. We outline a strategy to systematically tune the band gap and valence and conduction band positions of metal halide perovskites through control of the cation composition. Using this strategy, we demonstrate solar cells that harvest light in the infrared up to $1040 \mathrm{~nm}$, reaching a stabilized power conversion efficiency of $17.8 \%$, showing promise for improvements of the bottom cell of all-perovskite tandem solar cells. The mechanisms of cation-based band gap tuning we describe are broadly applicable to 3D metal halide perovskites, and will be useful in further development of perovskite semiconductors for optoelectronic applications.
\end{abstract}

\section{Introduction}

Metal halide perovskites of the composition $\mathrm{AMX}_{3}$, where $\mathrm{M}$ is a metal and $\mathrm{X}$ is a halide, are a promising class of materials for highly efficient solar cells ${ }^{1-4}$ that can be fabricated by inexpensive and scalable methods ${ }^{5-8}$. One of the most attractive features of perovskites for 
photovoltaics is the ability to tune their band gap by simple compositional substitution. For example, the band gap of methylammonium lead iodide, the prototypical perovskite photovoltaic absorber, can be tuned continuously from 1.6 to $2.3 \mathrm{eV}^{9}$ by replacement of varying fractions of iodine with bromine. A similar strategy allows the band gap of formamidinium lead trihalide to be tuned from 1.48 to $2.23 \mathrm{eV}^{10}$. As a result, compositional band gap tuning enables metal halide perovskites to be optimized for use as either top or bottom cell absorber in a tandem solar cell, or as the sole absorber in a single junction solar cell.

The perovskite materials used in the best performing solar cells to date have largely used materials with band gaps in the range of $1.48-1.62 \mathrm{eV}^{1,2,10,11}$. To harvest a wider range of the solar spectrum, however, materials with smaller band gaps are necessary. This is especially essential to make efficient tandem solar cells, which offer the possibility of surpassing the fundamental Shockley-Queisser limit on the efficiency of a single junction solar cell ${ }^{12}$. Allperovskite tandem solar cells offer the possibility of low-temperature and scalable fabrication of highly efficient solar cells on lightweight and flexible substrates ${ }^{3,7}$. However, simple halide substitution does not offer a way to reduce the band gap below $1.48 \mathrm{eV}$. To achieve lower band gaps, strategies that substitute the cations have been used - most successfully, substitution of the lead cation on the B-site with $\operatorname{tin}^{3,13-16}$. Based on this strategy, we recently demonstrated a tinlead perovskite absorber as the bottom cell in monolithic all-perovskite tandem solar cells with close-to-optimal band gaps ${ }^{3}$. In addition, for top cells of tandems, although a wide range of larger band gaps is accessible through partial halide substitution, many of these blends are not photostable, and show the Hoke effect, or segregation into bromide-rich and iodide-rich domains upon exposure to light ${ }^{17,18}$. This effect makes it difficult for many high-gap materials to achieve high open circuit voltages in solar cells. As a result, alternate strategies of band gap tuning are important for wide-gap perovskite materials as well.

In this report, we investigate the mechanisms by which the A-site cation can tune the band gap. Computation has predicted ${ }^{19,20}$, and experiments have confirmed ${ }^{21,22}$, that using smaller cations on the A-site increases the band gap of lead halide perovskites. This is manifested in increased bandgaps as the $\mathrm{Cs}$ content in $\mathrm{FA}_{1-\mathrm{x}} \mathrm{Cs}_{\mathrm{x}} \mathrm{PbI}_{3}$ perovskites is increased. Here, we report that tin iodide perovskites $\left(\mathrm{FA}_{1-\mathrm{x}} \mathrm{Cs}_{\mathrm{x}} \mathrm{SnI}_{3}\right)$ show the opposite effect - substituting the large organic formamidinium cation with the smaller cesium steadily decreases their band gap. Using synchrotron X-ray Diffraction along with X-ray and Ultraviolet Photoelectron Spectroscopy measurements, we identify the mechanism by which the effect is produced, and how it differs from the effect of Cs substitution on lead-based perovskites. We conclude that the A-site cation influences the extent of metal-halide orbital overlap. This change in metal-halide bonding has a direct impact on valence and conduction band positions and the band gap, and may impact other important properties such as stability of the perovskite lattice ${ }^{23}$.

An anomalous band gap bowing has been reported for mixed tin-lead halide perovskites, where the lowest band gap is found not for the pure tin or pure lead halide materials, but for an intermediate composition ${ }^{24}$. By combining the strategies of A-site and B-site substitution, we show systematic tuning of the band gap in mixed tin-lead perovskites from 1.24 to $1.58 \mathrm{eV}$. Having mapped band gaps across a wide range of $\mathrm{FA}-\mathrm{Cs}$ and $\mathrm{Sn}-\mathrm{Pb}$ perovskite compositions, we select the compositions with the smallest band gaps, which are closest to ideal for the bottom cell of a tandem, and fabricate solar cells with them, demonstrating single junction solar cells with 
efficiency above $14 \%$. The external quantum efficiency (EQE) spectra show systematic shifts of the spectrum onset to lower energies, resulting in improvements to the short-circuit current. Our results shed light on the mechanism of cation-based band gap tuning in metal halide perovskites and have direct relevance to the development of tandem solar cells with optimized band gaps.

\section{Results and Discussion}

\section{Band Gap Tuning by A-site Cation Composition}
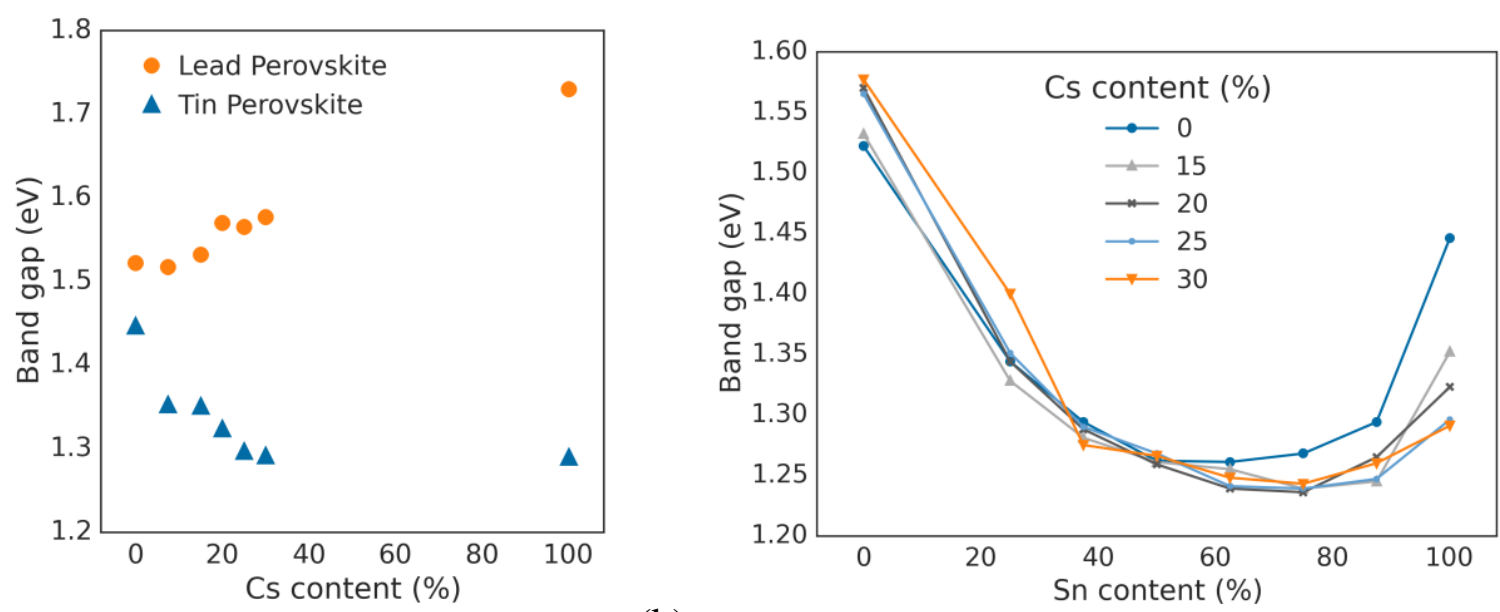

(a)

(b)

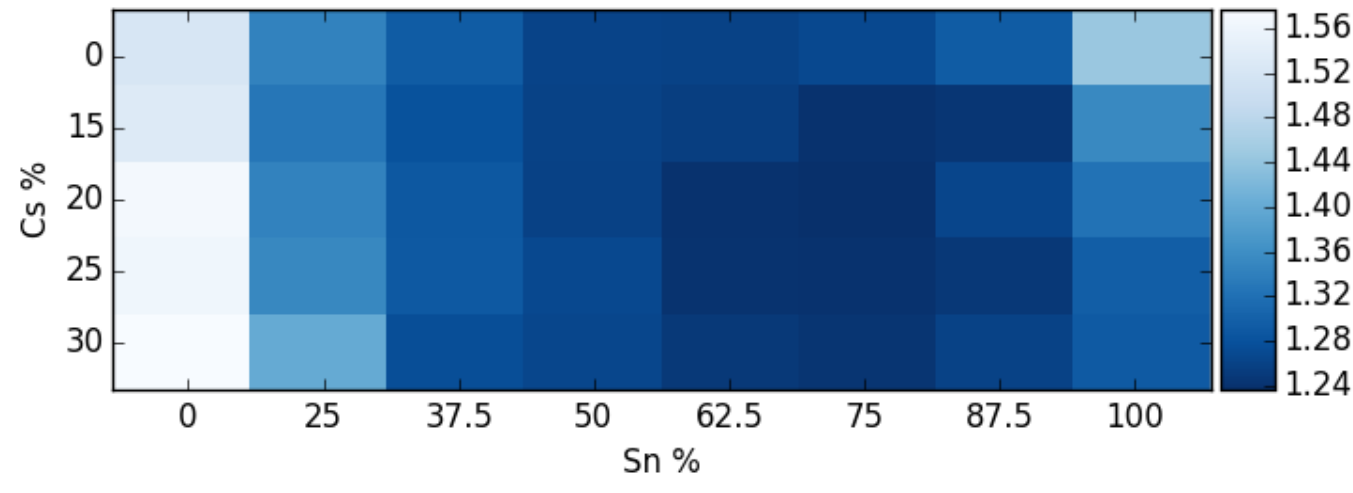

(c)

Figure 1: (a) Optical band gap of lead- and tin-based perovskites $\mathrm{FA}_{1-\mathrm{x}} \mathrm{Cs}_{\mathrm{x}} \mathrm{MI}_{3}(\mathrm{M}=\mathrm{Sn}, \mathrm{Pb})$ as a function of cesium content. (b) Band gap of five series of tin-lead mixed perovskites, corresponding to cesium fractions of $0,15,20,25$, and 30\%. (c) 2-dimensional map of band gap (in $\mathrm{eV}$ ) across the formamidinium-cesium and tin-lead compositional space studied.

Figure 1 shows experimentally measured optical band gaps for series of tin and lead perovskites as a function of A-site cation composition, which we vary from pure formamidinium (FA) to mixes of FA with cesium (Cs). To measure the band gap, we conduct sensitive measurements of absorption spectra in an integrating sphere and fit the onsets using Tauc plots. Measurement in an integrating sphere rather than using a simple transmission measurement is important for series of films whose roughness or haze varies widely, since light scattering can cause varying errors in measured absorption spectra. Fig. 1a depicts the effect of substituting FA with Cs for lead halide 
and tin halide perovskites. As previous theoretical ${ }^{19}$ and experimental ${ }^{21,22}$ work has shown, the lead halide compounds show an increase in bandgap as Cs is substituted for FA. In contrast, for the tin halide perovskites, the bandgap undergoes a dramatic reduction as the Cs content is increased.

Combining the effects of A-site cation size described above with the previously reported band gap bowing in mixed tin-lead perovskites, we demonstrate systematic tuning of the band gap between $1.24 \mathrm{eV}$ and $1.58 \mathrm{eV}^{25}$. Figure $1 \mathrm{~b}$ and $\mathrm{c}$ show maps of the optical band gap across a wide range of $\mathrm{A}$-site and $\mathrm{B}$-site cation compositions. We vary the A-site cation from pure formamidinium to a mix with $30 \%$ cesium, and the B-site cation between pure lead and pure tin. Each series of tin-lead perovskites shows the band gap bowing trend that has been reported for methylammonium and formamidinium tin-lead iodide perovskites ${ }^{3,13}$. The addition of cesium shifts the band gaps of the tin-rich compositions to lower values, consistent with the trend of cesium lowering the band gap of the pure tin iodide perovskite. We did not use cesium contents higher than $30 \%$ as they resulted in non-uniform films and the band gap reduction of FA ${ }_{x} \mathrm{Cs}_{\mathrm{x}} \mathrm{SnI}_{3}$ appears to have levelled off with around $30 \% \mathrm{Cs}$ substitution. The Goldschmidt tolerance factor across most of the compositional range studied here (Figure S1) is close to 1, consistent with these compositions being stable in a perovskite phase.

\section{Effects of Lattice Distortions}




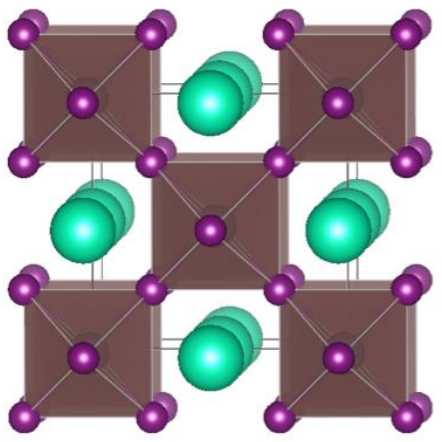

Lattice Contraction

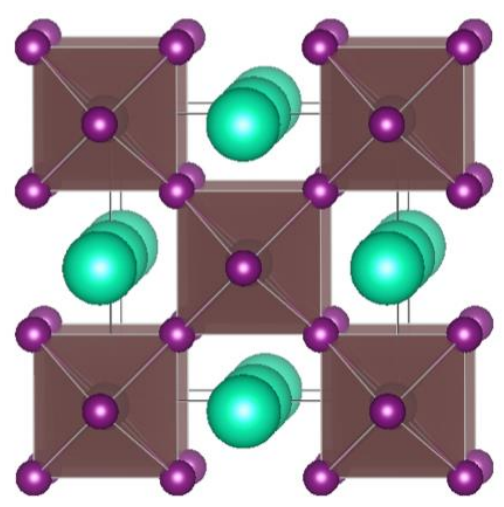

\section{Lattice Contraction}

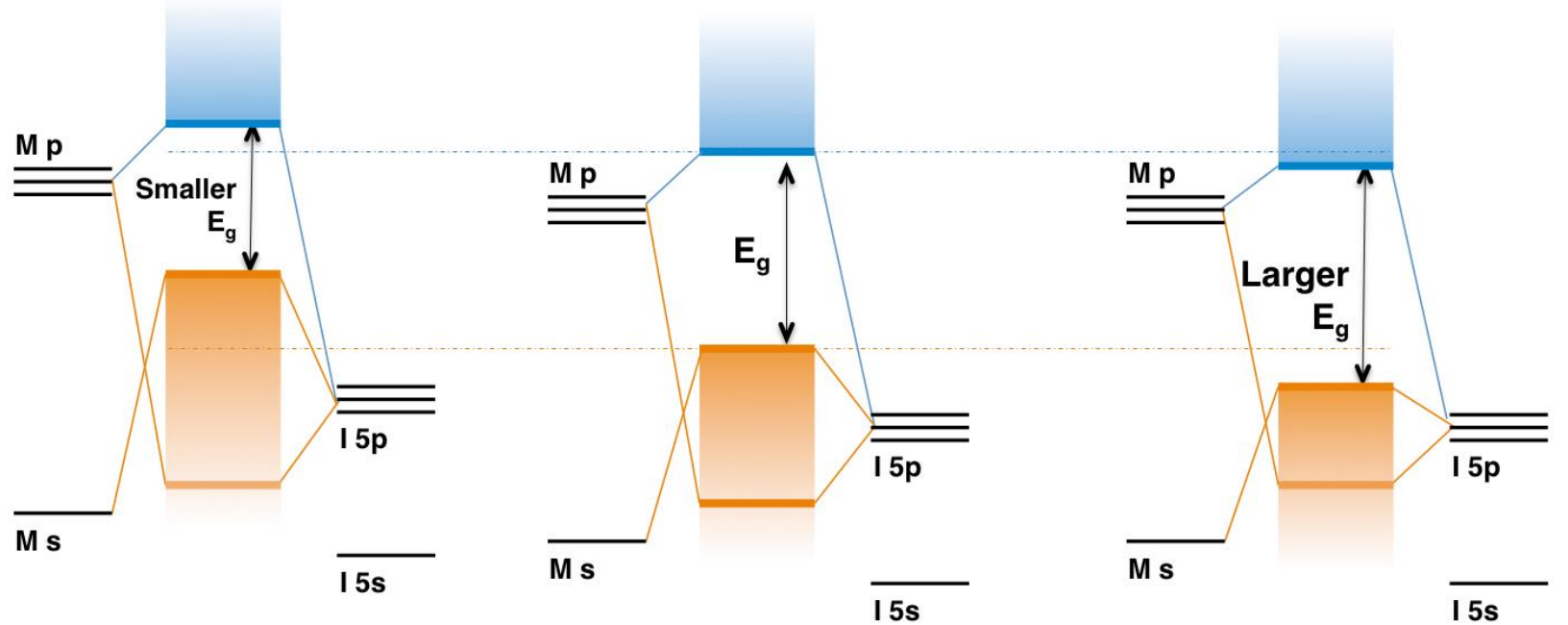

Figure 2: Perovskite lattice diagrams - undistorted cubic (center panel), with lattice contraction (left panel) and with octahedral tilting (right panel) ${ }^{26}$. Below each lattice is a schematic energy level diagram, showing how each kind of distortion affects the valence and conduction bands 27,28 .

First principles electronic structure calculations have shown that the valence band maximum of $\mathrm{ABX}_{3}$-type metal halide perovskites is an antibonding hybrid state of the metal $\mathrm{s}$ and halide $\mathrm{p}$ orbitals, while the conduction band minimum is a hybrid of metal $\mathrm{p}$ and halide $\mathrm{p}$ orbitals with less antibonding and more nonbonding character (Figure 2) ${ }^{27-29}$. Since the valence band maximum is composed of antibonding combinations of metal $\mathrm{s}$ and halogen $\mathrm{p}$ orbitals ${ }^{27}$, any change to the perovskite lattice that increases the amount of M-X overlap will destabilize the valence band, raising it in energy. The conduction band, derived from p-orbitals and having poorer orbital overlap, is expected to respond less strongly to distortions in lattice structure than the valence band that is derived from metal s-orbitals ${ }^{30}$. In other words, the conduction band minimum is closer to a nonbonding hybrid than the antibonding valence band maximum, and hence will shift by a smaller amount than the valence band maximum upon introducing a structural change. As a result, structural changes that increase M-X overlap will decrease the band gap, while structural changes that reduce metal-halide overlap will increase the band gap. This is in line with computational studies of the effect of strain on the positions of the valence 
and conduction bands, which show that the band gap decreases as the lattice contracts isotropically due to a shift to higher energies predominantly of the valence band ${ }^{29}$.

Since we expect the M-X overlap to be the main factor dictating changes in bandgap, we perform synchrotron X-Ray Diffraction (XRD) measurements to identify structural differences due to changing the A-site cation that might influence orbital overlap. We record XRD patterns of tin and lead iodide perovskites, with 0,15 , and $25 \%$ substitution of cesium for formamidinium at the A site. Consistent with previous reports ${ }^{31}$, the XRD pattern of $\mathrm{FAPbI}_{3}$ (Figure 3a) reflects a cubic perovskite, which we index in the space group $\operatorname{Pm} \overline{3} \mathrm{~m}$. Upon partial Cs substitution, additional superlattice peaks appear in the XRD patterns (Figure 3b) at positions corresponding to halfintegral values of Miller indices, indicating a tetragonal distortion and a lowering of symmetry by tilting of the $\mathrm{PbI}_{6}$ octahedra that make up the perovskite lattice. We index the structures of $\mathrm{FA}_{0.85} \mathrm{Cs}_{0.15} \mathrm{PbI}_{3}$ and $\mathrm{FA}_{0.75} \mathrm{Cs}_{0.25} \mathrm{PbI}_{3}$, in the tetragonal space group $P 4 / \mathrm{mbm}$. This tetragonal distortion implies a reduction of the metal-halide-metal bond angle below the value of 180 degrees that exists in a cubic structure. Since the metal-halide orbital overlap is a $\sigma^{*}$ interaction, reduction of the M-X-M bond angle reduces the extent of overlap between metal and halide orbitals, competing with any effects of reduction in bond length. The net result is that the valence band moves to slightly lower energies as Cs substitution is increased, widening the band gap, as confirmed by the XPS and UPS data in Figure 4a. This trend is in line with experimental investigations by Mitzi et al for layered perovskites ${ }^{32}$ and theoretical predictions by Giustino et al for $3 \mathrm{D}$ hybrid perovskites ${ }^{19}$, which show that reduction in the $\mathrm{M}-\mathrm{X}-\mathrm{M}$ bond angle produces an increase in the band gap.

The XRD patterns in Figure $3 \mathrm{~b}$ show that tin-based perovskites $\left(\mathrm{FA}_{1-\mathrm{x}} \mathrm{Cs}_{\mathrm{x}} \mathrm{SnI}_{3}\right)$ retain cubic symmetry upon partial cesium substitution, in contrast to the lead-based perovskites. The tin perovskite shows no octahedral tilting, which would be apparent by superlattice peaks between the cubic perovskite peaks (near $q=1.57 \AA^{-1}$ ). This is different from the behaviour of the lead halide perovksites, where the octahedra are forced to tilt to compensate for the reduced A-site cation size. Instead, cesium substitution only causes a decrease in the cubic lattice parameter of $\mathrm{FA}_{1-\mathrm{x}} \mathrm{Cs}_{\mathrm{x}} \mathrm{SnI}_{3}$. Since the lattice parameter corresponds to twice the $\mathrm{Sn}$-I bond length, this implies a contraction of the Sn-I bond, resulting in an increase in metal-halide orbital overlap. As outlined above, this increased orbital overlap shifts the antibonding valence band to higher energies and lowers the band gap. Measurement of the valence band position by X-ray Photoelectron Spectroscopy (XPS) confirms this shift to higher energies upon Cs substitution (Figure 4b).

Figure 4 also shows that the band positions are significantly shifted upon Cs-substitution, especially for tin perovskites. This has implications for tuning the energy levels of electron and hole contacts used in a perovskite solar cell, due to the requirement that the valence band of a hole transport material must be shallower than that of the perovskite, and the conduction band of the electron transport material must be deeper than that of the perovskite, to prevent formation of an energetic barrier to charge extraction ${ }^{33}$.

Incidentally, the octahedral tilting we observe in thin films of $\mathrm{FA}_{1-\mathrm{x}} \mathrm{Cs}_{\mathrm{x}} \mathrm{PbI}_{3}$ is different from that reported for methylammonium lead iodide. In both cases, tilting is around the c-axis. In $\mathrm{MAPbI}_{3}$, these tilts are out-of-phase along the c-axis, i.e.: adjacent octahedra along the c-axis are tilted in 
opposite directions. In Glazer notation ${ }^{34}$ this is denoted $a^{0} a^{0} c^{-}$, and this tilt system uniquely corresponds to the space group $\mathrm{I} 4 / \mathrm{mcm}^{35,36}$. The indexing of $\mathrm{FA}_{1-\mathrm{x}} \mathrm{Cs}_{\mathrm{x}} \mathrm{PbI}_{3}$ in space group $\mathrm{P} 4 / \mathrm{mbm}$, however, implies that adjacent octahedra are tilted in the same direction about the caxis, or $a^{0} a^{0} c^{+}$in Glazer notation. $\mathrm{FAPbI}_{3}$, without any $\mathrm{Cs}$ substitution, has been shown to transition from the cubic perovskite to the tetragonal $\mathrm{P} 4 / \mathrm{mbm}$ perovskite structure at $285 \mathrm{~K}$, i.e.

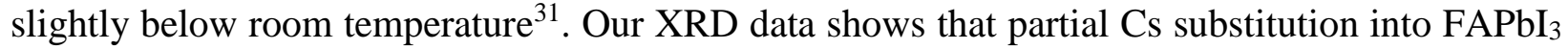
induces the lattice to adopt the same P4/mbm tetragonal structure at room temperature.

In summary, although the energy levels of the A-site cation are deep within the bands and do not directly dictate the frontier energy levels ${ }^{19}$, the A-site cation indirectly influences the band positions and band gap through two competing effects. Lattice contraction increases metal-halide orbital overlap, which raises the bands to shallower energies and decreases the band gap. Octahedral tilting reduces metal-halide orbital overlap, moving the bands to deeper energies and increasing the band gap. Our detailed analysis was only performed on the pure tin-halide and lead-halide perovskites, for simplicity, but our results in Figure 1 demonstrate that this logic can be extended to the mixed metal compounds. Those with high tin content $(>50 \%)$ show smaller bandgaps with increased Cs content, while those with low tin content $(<50 \%)$ have larger bandgaps as the Cs content is increased. The XPS and UPS data in Figure 4 shows that in addition to influencing the band gap, the cation can also alter the absolute energetic positions of the bands, which will be relevant to selecting materials with appropriate energy levels to accept either electrons or holes from the perovskite in a solar cell.

(a)

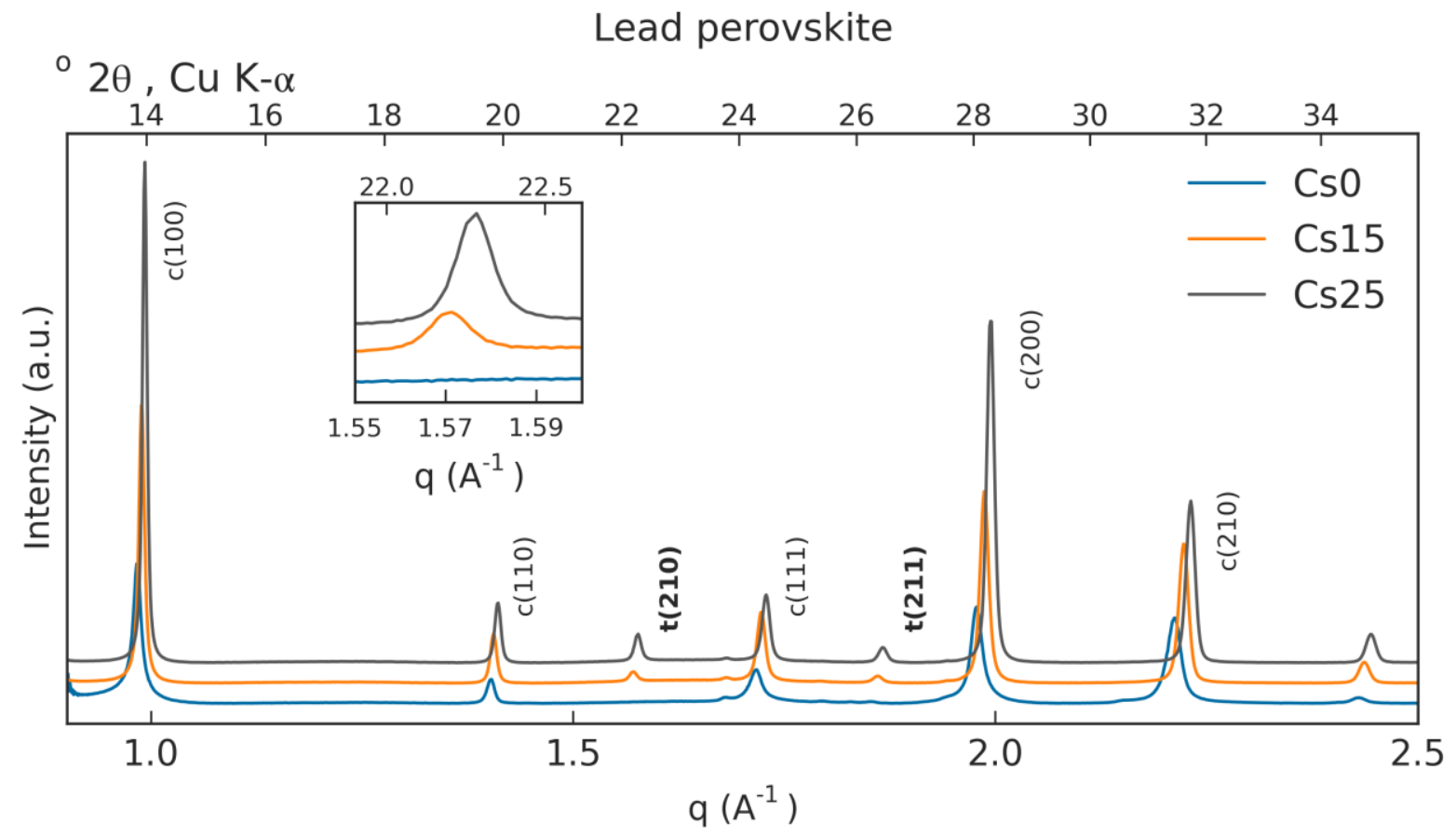


(b)
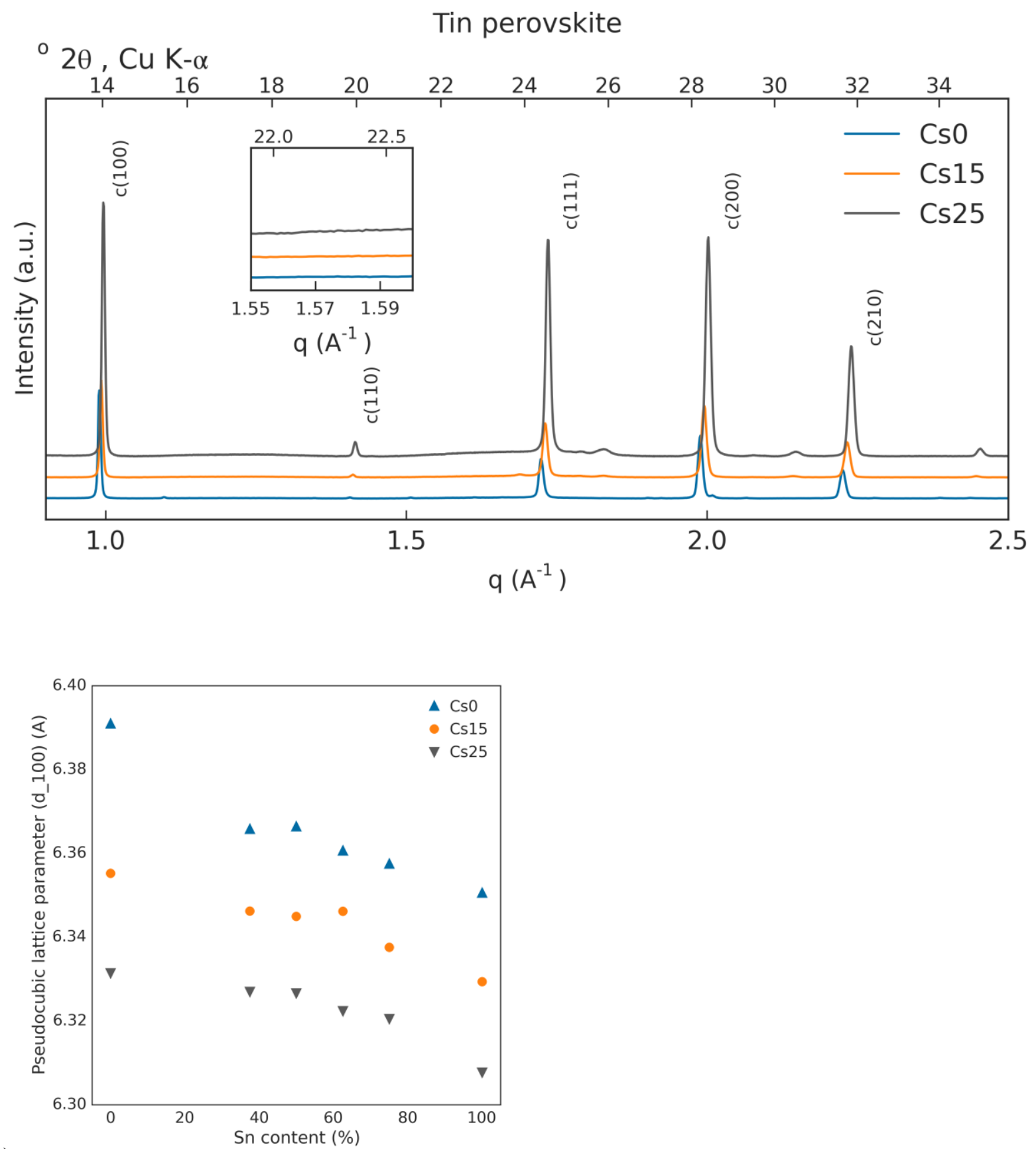

(c)

Figure 3: X-ray diffraction patterns for (a) lead perovskite and (b) tin perovskite with varying cesium substitution. XRD patterns are plotted with respect to $\mathrm{q}$, which is equivalent to $2 * \pi / \mathrm{d}$ where $\mathrm{d}$ is the $\mathrm{d}$-spacing of the diffracting set of planes. In addition, we plot with respect to 2 theta values ( $\mathrm{Cu} \mathrm{K \alpha}$ radiation) for easy comparison with other published data. The insets show zoomed in versions of the lead- and tin-based perovskite patterns around $q=1.57 \AA^{-1}$, showing the presence of a superlattice peak only in the lead-based perovskite. The small peaks in the tin perovskite pattern with $\mathrm{Cs}$ substitution at $\mathrm{q}=1.82 \AA^{-1}$ and $2.15 \AA^{-1}$ correspond to a non- 
perovskite impurity phase as we show in Figure S3. (c) Pseudocubic lattice parameter as a function of cation composition.

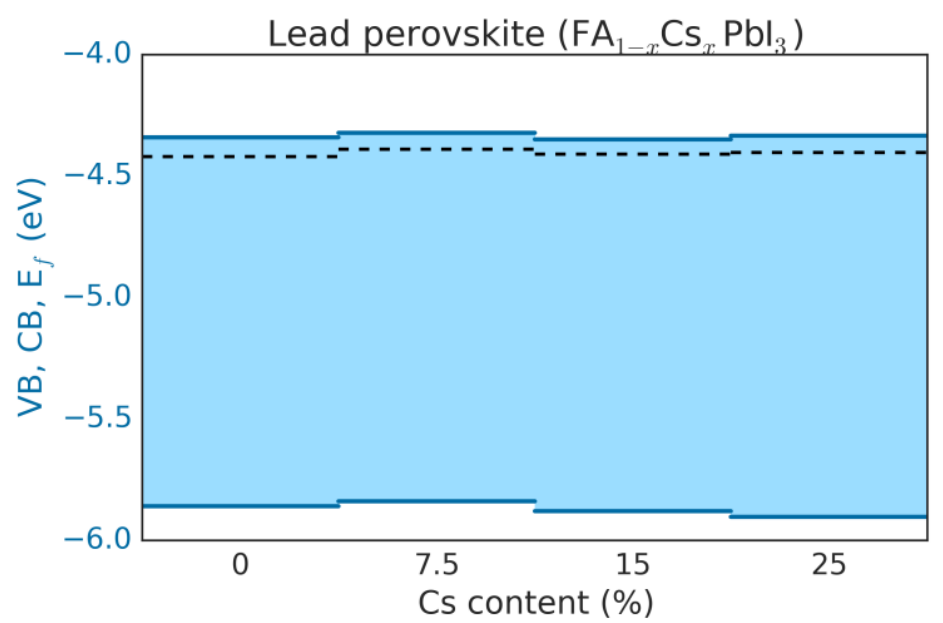

(a)

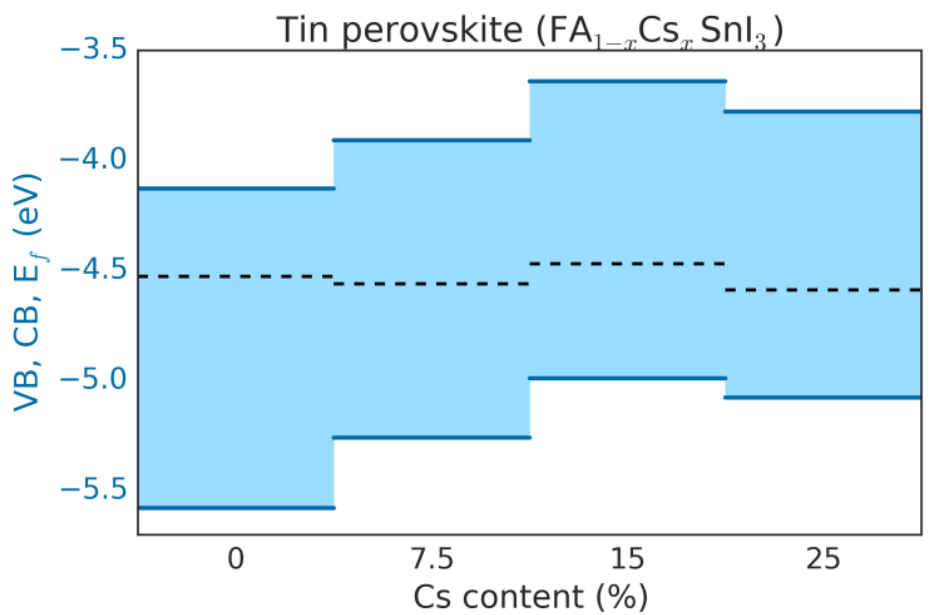

(b)

Figure 4: Positions of the valence and conduction bands (solid lines) and Fermi levels (dashed lines) for (a) lead perovskites and (b) tin perovskites, as functions of Cs substitution. Valence band maxima and Fermi levels were measured by XPS and UPS respectively, and conduction band minima were inferred by adding the measured optical band gap to the valence band maxima.

\section{Solar Cells with Low Band Gaps}

For tandem solar cells comprising two junctions, the ideal band gap for the bottom cell is around 1.1 to $1.2 \mathrm{eV}^{37}$. Based on this criterion, the most promising perovskite compositions for low gap cells in tandems among the compositions studied above have Cs content around $20 \%$, with Sn contents between $50 \%$ and $75 \%$. We fabricate solar cells within this compositional space and find that most compositions in this range can form efficient solar cells with up to $14 \%$ power conversion efficiency (Figure 5a). Importantly, the external quantum efficiency onsets of the 
solar cells follow the same trend as the bandgaps derived from absorption measurements. The EQE spectra for some selected tin-rich compositions with cesium are shown in Figure 5b. As they indicate, cesium substitution at the A-site allows the tin-rich compositions to access low band gaps - solar cells made with perovskites using $20 \%$ cesium and $75 \%$ Sn harvest light out to $1040 \mathrm{~nm}, 40 \mathrm{~nm}$ further into the near infrared than for the low gap material that we have previously used in monolithic all-perovskite tandems ${ }^{3}$.

More broadly, this study identifies a guideline for achieving small band gaps with tin-lead perovskites - which is to select a cubic perovskite with a small pseudocubic lattice parameter. While the compounds with formamidinium and cesium at the A-site that we have selected for this study successfully achieve this, they suffer from short PL lifetimes of under $1 \mathrm{~ns}^{3}$, which could lead to poor carrier extraction from perovskite layers thick enough to absorb most of the incident light. Zhao et al have recently reported a different tin-rich composition that contains methylammonium, $\mathrm{MA}_{0.4} \mathrm{FA}_{0.6} \mathrm{Sn}_{0.6} \mathrm{~Pb}_{0.4} \mathrm{I}_{3}$, to have long carrier lifetimes of around $250 \mathrm{~ns}^{15}$. As we show using X-ray Diffraction in Figure S6, this composition has the desirable characteristics of a contracted lattice with cubic symmetry maintained, that leads to a small band gap of 1.24 $\mathrm{eV}$. With this composition, we demonstrate a solar cell with a stabilized power conversion efficiency of $17.8 \%$ (Figure $5 \mathrm{c}$ ). The high $\mathrm{V}_{\mathrm{oc}}$ relative to band gap also demonstrates that these compositions all show small losses from the band gap to the open circuit voltage $\left(\mathrm{E}_{\mathrm{g}}-\mathrm{qV}_{\mathrm{oc}}\right.$ around $0.47 \mathrm{~V}$ for the FA-Cs compositions, and merely $0.41 \mathrm{~V}$ for the MA-containing composition), as previously demonstrated for tin-lead perovskite solar cells ${ }^{3,38}$. There is still room for improvement, however in the short-circuit current harvested - as the EQE curves (Figures 5b and 5d) suggest, the solar cells suffer from relatively low EQE in the infrared, and will likely benefit from having thicker perovskite layers to absorb most of the above-bandgap light. Since the EQE curves also show periodic dips that suggest loss of light by reflection, the short-circuit current could also be raised by antireflective coatings or scattering layers on the light-facing surface of the cell ${ }^{39}$. 


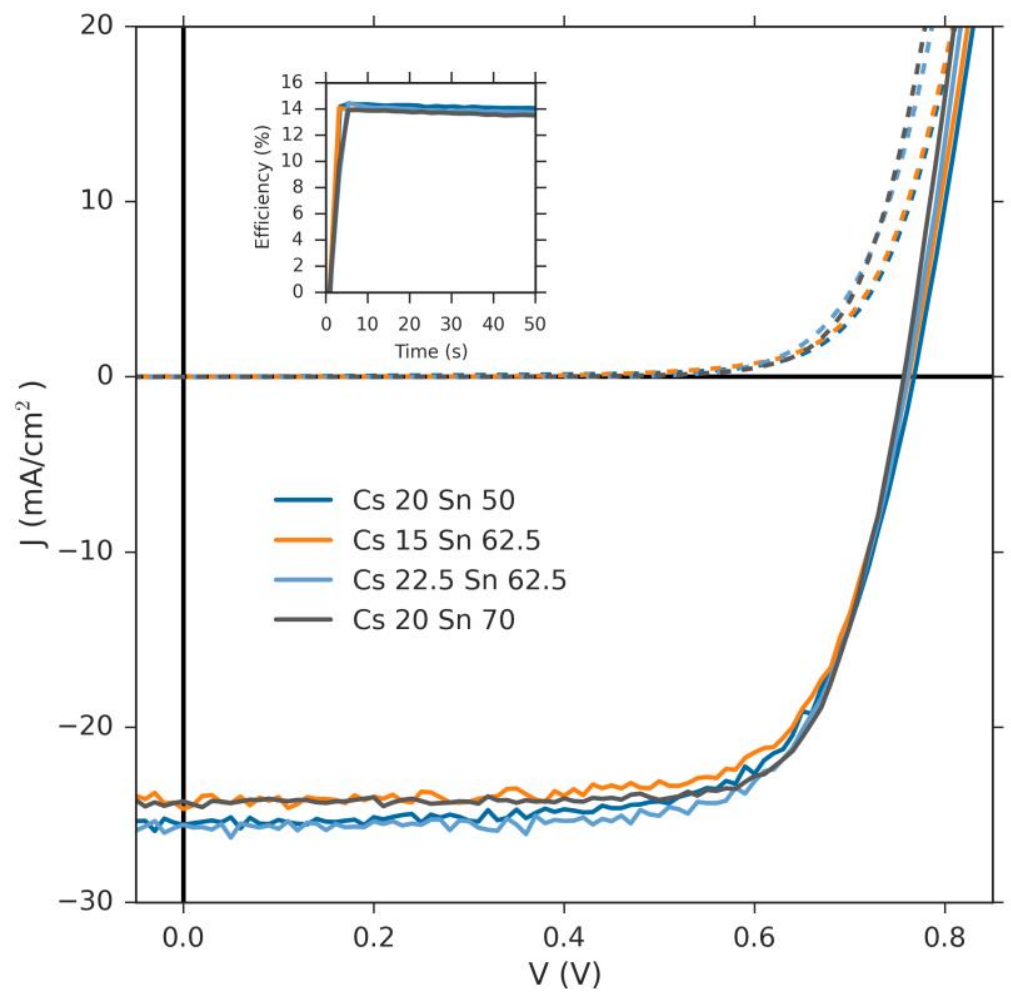

(a)

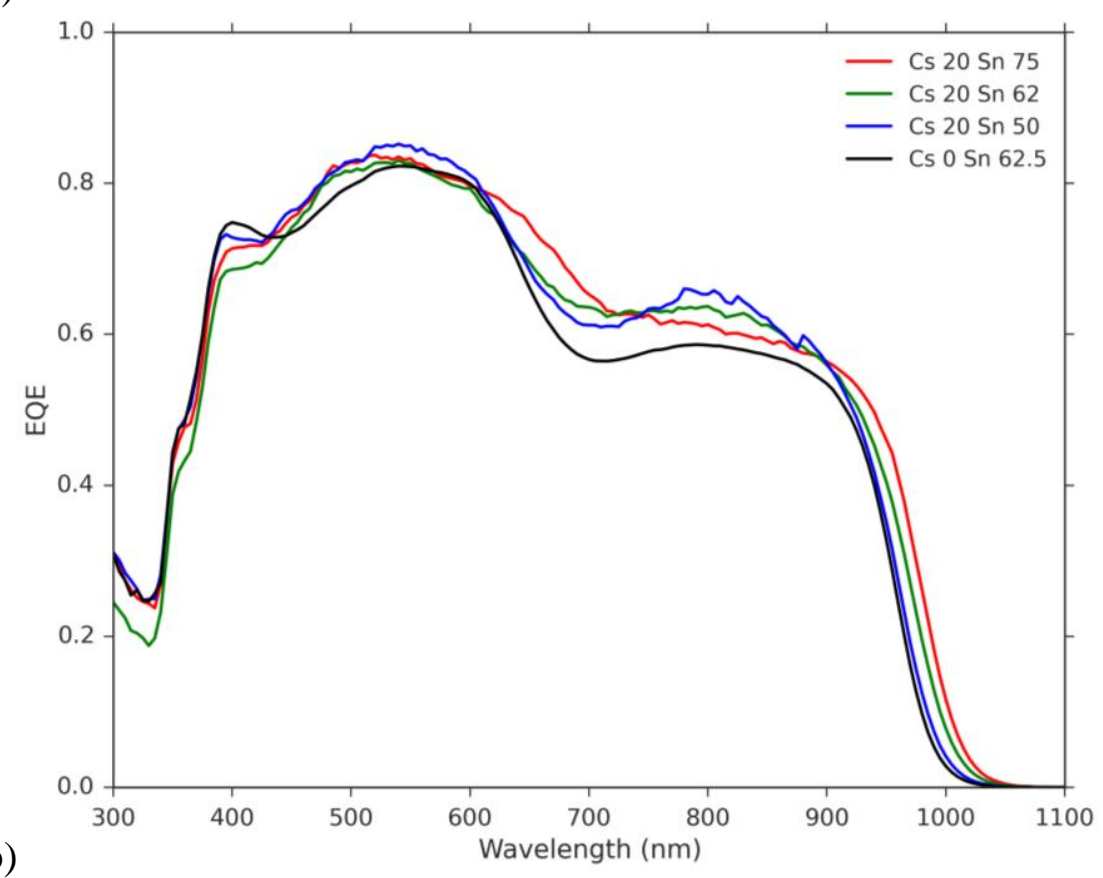




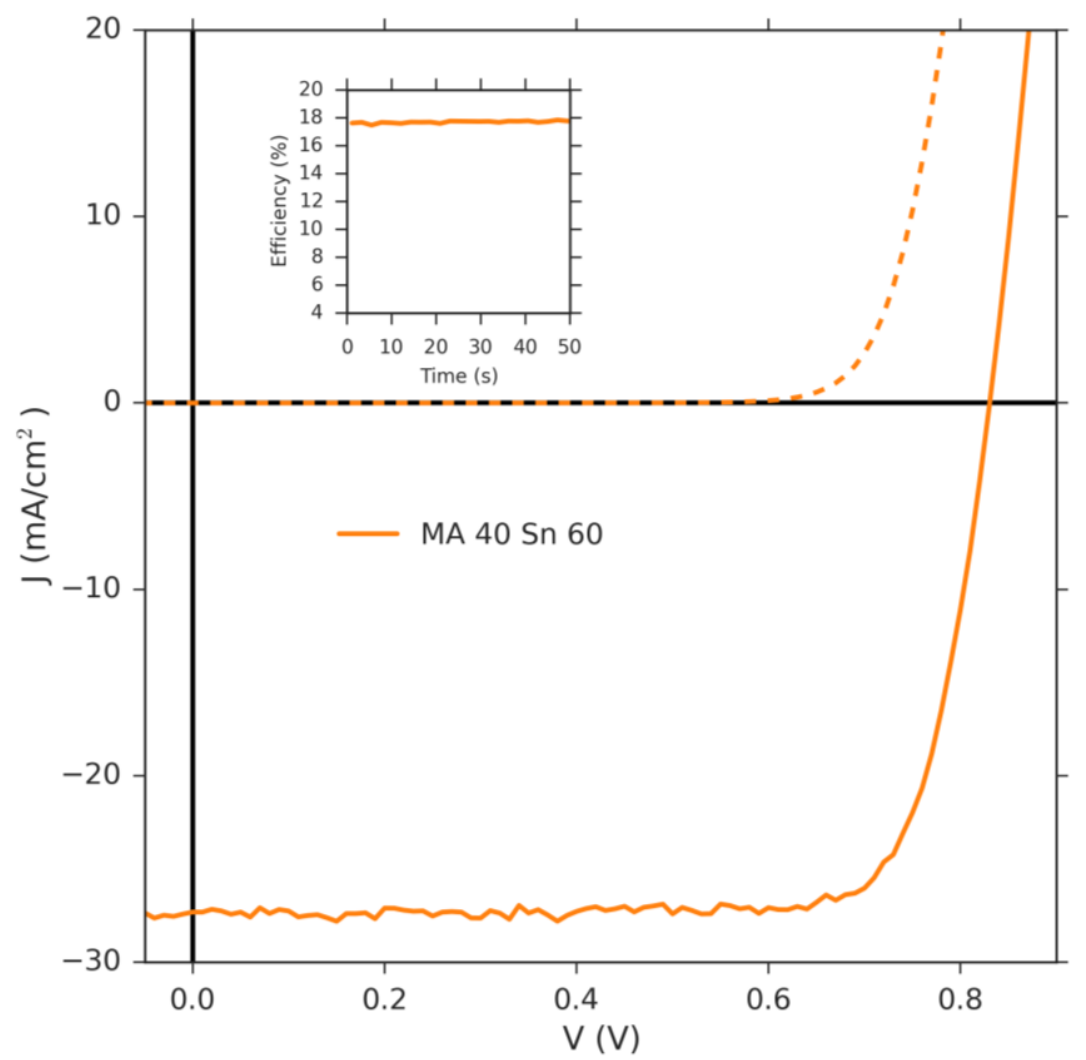

(c)

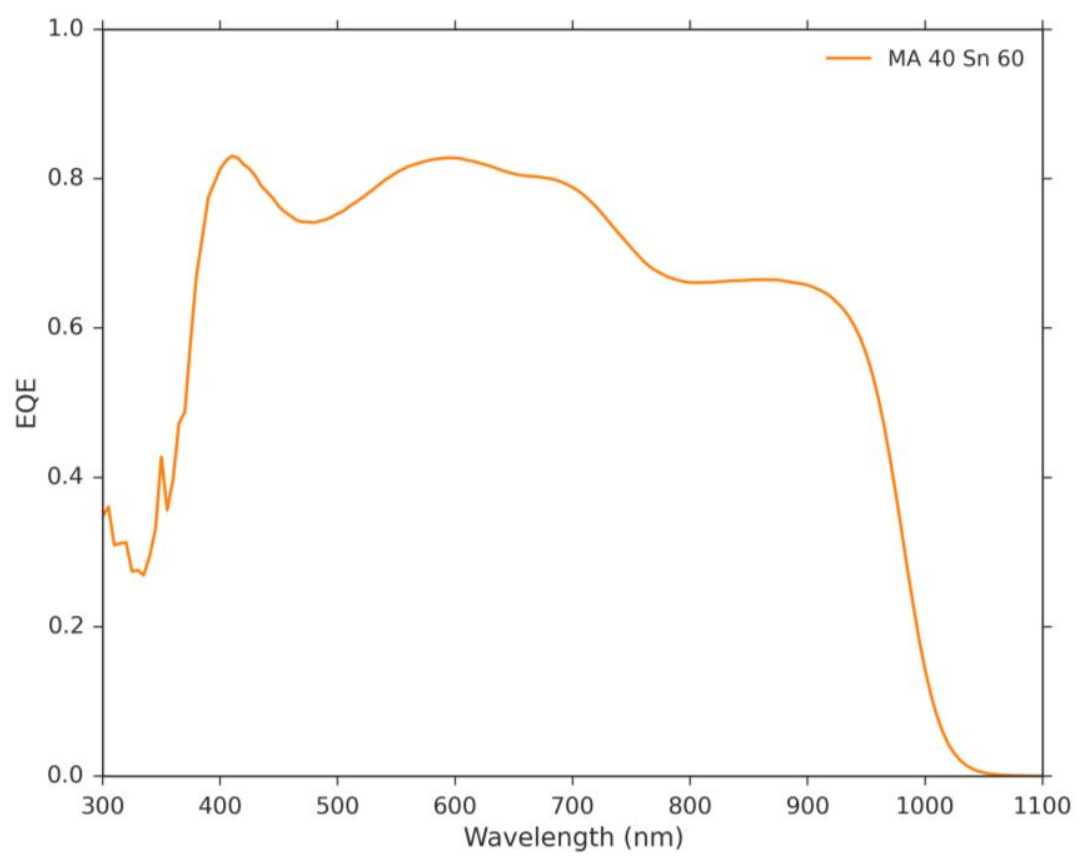

Figure 5 (a) JV curves of solar cells with a few high-performing compositions $\left(\mathrm{FA}_{1-\mathrm{x}} \mathrm{Cs}_{\mathrm{x}} \mathrm{Sn}_{\mathrm{y}} \mathrm{Pb}_{1-}\right.$ $\mathrm{y}_{3}$ ), with stabilized maximum power point tracking curves in the inset. Solid lines denote JV curves under simulated AM1.5G sunlight and dashed lines denote JV curves in the dark. (b) EQE spectra of devices made with perovskites with $50-75 \%$ tin and with Cs substitution, showing redshifted onsets. EQE spectrum of a $62.5 \%$ tin device without Cs substitution is included for 
comparison. (c), (d) JV, stabilized maximum power point tracking, and EQE curves of a solar cell based on the perovskite composition $\mathrm{MA}_{0.4} \mathrm{FA}_{0.6} \mathrm{Sn}_{0.6} \mathrm{~Pb}_{0.4} \mathrm{I}_{3}$

\section{Conclusion and Outlook}

Future work aimed at further decreasing the band gap of metal halide perovskites could focus on using other small A-site cations or even partially substituting smaller ions other than tin and lead onto the B-site to shrink the lattice parameter, as long as the compound retains a cubic crystal structure. Future studies must also address the question of why octahedral tilting occurs upon partial Cs-substitution for lead perovskites but not for tin perovskites. A recent computational study has shown that tilting in metal halide perovskites occurs due to a second order Jahn-Teller effect - octahedral tilting stabilizes the hybrid orbitals that form states deep in the valence band, but the size of the A-site cation dictates whether this distortion is sterically favourable ${ }^{28}$. Interestingly, the authors of that study observe that substituting the lead at the B-site with calcium, strontium, or barium increases the propensity to tilt, hinting at the importance of the nature of the B-site cation in determining whether the lattice shows octahedral tilting. The study also finds that in some perovskites, hydrogen bonding by the A-site cations can stabilize a tilted structure.

The competing effects of octahedral tilt and lattice contraction, combined with the band gap bowing across a tin-lead compositional range, provide a strategy to systematically tune band gaps using substitution of the A-site and B-site cations. The band gaps achieved through this compositional tuning are close to ideal for being used in the bottom cell of a tandem solar cell, harvesting light out to around $1040 \mathrm{~nm}$ in the solar spectrum. The effects of cation compositional tuning on the band gap that are outlined here are general and should apply to other families of metal halide perovskites, providing a general framework of design rules for achieving a desired band gap and band positions with a perovskite semiconductor.

\section{Acknowledgements}

We thank Kevin A. Bush and Kyle Frohna for productive and stimulating conversations, Adam H. Slavney and David A. Hanifi for insightful comments on the manuscript, and Caleb C. Boyd for assistance with solar cell fabrication. This research was supported by the Office of Naval Research, U.S. Department of Defense. Use of the Stanford Synchrotron Radiation Lightsource, SLAC National Accelerator Laboratory, is supported by the U.S. Department of Energy, Office of Science, Office of Basic Energy Sciences under Contract No. DE-AC02-76SF00515. TL is funded by a Marie Sklodowska Curie International Fellowship under grant agreement H2O2IFGA-2015-659225. A.G. is supported by NSF GRFP (DGE-1147470). B.C. is a postdoctoral research fellow of the Research Fund Flanders (FWO). A.B. is a PhD fellow of FWO.

\section{Supporting Information}

Experimental information, tolerance factor map across the compositional space studied, additional XRD information, UPS and XPS spectra, and solar cell performance data. 


\section{References}

(1) Yang, W. S.; Noh, J. H.; Jeon, N. J.; Kim, Y. C.; Ryu, S.; Seo, J.; Seok, S. I. Science (80-. ). 2015, 348 (6240), 1234.

(2) Saliba, M.; Matsui, T.; Seo, J.-Y.; Domanski, K.; Correa-Baena, J.-P.; Nazeeruddin, M. K.; Zakeeruddin, S. M.; Tress, W.; Abate, A.; Hagfeldt, A.; Grätzel, M. Energy Environ. Sci. 2016, 9 (6), 1989.

(3) Eperon, G. E.; Leijtens, T.; Bush, K. A.; Prasanna, R.; Green, T.; Wang, J. T.; McMeekin, D. P.; Volonakis, G.; Milot, R. L.; May, R.; Palmstrom, A.; Slotcavage, D. J.; Belisle, R. A.; Patel, J. B.; Parrott, E. S.; Sutton, R. J.; Ma, W.; Moghadam, F.; Conings, B.;

Babayigit, A.; Boyen, H.; Bent, S.; Giustino, F.; Herz, L. M.; Johnston, M. B.; McGehee, M. D.; Snaith, H. J. Science (80-. ). 2016, 354 (6314), 861.

(4) Bush, K. A.; Palmstrom, A. F.; Yu, Z. J.; Boccard, M.; Cheacharoen, R.; Mailoa, J. P.; McMeekin, D. P.; Hoye, R. L. Z.; Bailie, C. D.; Leijtens, T.; Peters, I. M.; Minichetti, M. C.; Rolston, N.; Prasanna, R.; Sofia, S.; Harwood, D.; Ma, W.; Moghadam, F.; Snaith, H. J.; Buonassisi, T.; Holman, Z. C.; Bent, S. F.; McGehee, M. D. Nat. Energy 2017, 2 (4), 17009.

(5) Yang, M.; Li, Z.; Reese, M. O.; Reid, O. G.; Kim, D. H.; Siol, S.; Klein, T. R.; Yan, Y.; Berry, J. J.; van Hest, M. F. A. M.; others. Nat. Energy 2017, 2 (5), nenergy 201738.

(6) Tang, S.; Deng, Y.; Zheng, X.; Bai, Y.; Fang, Y.; Dong, Q. 2017, 1700302, 1.

(7) Forgács, D.; Gil-Escrig, L.; Pérez-Del-Rey, D.; Momblona, C.; Werner, J.; Niesen, B.; Ballif, C.; Sessolo, M.; Bolink, H. J. Adv. Energy Mater. 2016, 1602121.

(8) Hwang, K.; Jung, Y. S.; Heo, Y. J.; Scholes, F. H.; Watkins, S. E.; Subbiah, J.; Jones, D. J.; Kim, D. Y.; Vak, D. Adv. Mater. 2015, 27 (7), 1241.

(9) Noh, J. H.; Im, S. H.; Heo, J. H.; Mandal, T. N.; Seok, S. Il. Nano Lett. 2013, 13 (4), 1764.

(10) Eperon, G. E.; Stranks, S. D.; Menelaou, C.; Johnston, M. B.; Herz, L. M.; Snaith, H. J. Energy Environ. Sci. 2014, 7 (3), 982.

(11) Son, D.-Y.; Lee, J.-W.; Choi, Y. J.; Jang, I.-H.; Lee, S.; Yoo, P. J.; Shin, H.; Ahn, N.; Choi, M.; Kim, D.; Park, N.-G. Nat. Energy 2016, 1 (June), 16081.

(12) Shockley, W.; Queisser, H. J. J. Appl. Phys. 1961, 32 (3), 510.

(13) Im, J.; Stoumpos, C. C.; Jin, H.; Freeman, A. J.; Kanatzidis, M. G. J. Phys. Chem. Lett. 2015, 6 (17), 3503.

(14) Yang, Z.; Rajagopal, A.; Chueh, C. C.; Jo, S. B.; Liu, B.; Zhao, T.; Jen, A. K. Y. Adv. Mater. 2016, 28 (40), 8990.

(15) Zhao, D.; Yu, Y.; Wang, C.; Liao, W.; Shrestha, N.; Grice, C. R.; Cimaroli, A. J.; Guan, L.; Ellingson, R. J.; Zhu, K.; Zhao, X.; Xiong, R.-G.; Yan, Y. Nat. Energy 2017, 2 (March), 17018.

(16) Liao, W.; Zhao, D.; Yu, Y.; Shrestha, N.; Ghimire, K.; Grice, C. R.; Wang, C.; Xiao, Y.; Cimaroli, A. J.; Ellingson, R. J.; Podraza, N. J.; Zhu, K.; Xiong, R. G.; Yan, Y. J. Am. Chem. Soc. 2016, 138 (38), 12360.

(17) Hoke, E. T.; Slotcavage, D. J.; Dohner, E. R.; Bowring, A. R.; Karunadasa, H. I.; McGehee, M. D. Chem. Sci. 2015, 6 (1), 613.

(18) Slotcavage, D. J.; Karunadasa, H. I.; McGehee, M. D. ACS Energy Lett. 2016, 1 (6), 1199.

(19) Filip, M. R.; Eperon, G. E.; Snaith, H. J.; Giustino, F. Nat. Commun. 2014, 5, 5757.

(20) Amat, A.; Mosconi, E.; Ronca, E.; Quarti, C.; Umari, P.; Nazeeruddin, M. K.; Grätzel, M.; De Angelis, F. Nano Lett. 2014, 14 (6), 3608.

(21) Choi, H.; Jeong, J.; Kim, H. B.; Kim, S.; Walker, B.; Kim, G. H.; Kim, J. Y. Nano Energy 
2014, 7, 80 .

(22) McMeekin, D. P.; Sadoughi, G.; Rehman, W.; Eperon, G. E.; Saliba, M.; Horantner, M. T.; Haghighirad, A.; Sakai, N.; Korte, L.; Rech, B.; Johnston, M. B.; Herz, L. M.; Snaith, H. J. Science (80-. ). 2016, 351 (6269), 151.

(23) Leijtens, T.; Bush, K.; Cheacharoen, R.; Beal, R.; Bowring, A.; McGehee, M. D. J. Mater. Chem. A 2017, 0,1 .

(24) Hao, F.; Stoumpos, C. C.; Chang, R. P. H.; Kanatzidis, M. G. J. Am. Chem. Soc. 2014, 136 (22), 8094.

(25) Hao, F.; Stoumpos, C. C.; Chang, R. P. H.; Kanatzidis, M. G. J. Am. Chem. Soc. 2014, $136(22), 8094$.

(26) Momma, K.; Izumi, F. J. Appl. Crystallogr. 2011, 44 (6), 1272.

(27) Chang, Y. H.; Park, C. H.; Matsuishi, K. 2004, 44 (4), 889.

(28) Lee, J. H.; Bristowe, N. C.; Lee, J. H.; Lee, S. H.; Bristowe, P. D.; Cheetham, A. K.; Jang, H. M. Chem. Mater. 2016, 28 (12), 4259.

(29) Grote, C.; Berger, R. F. J. Phys. Chem. C 2015, 119 (40), 22832.

(30) Hoffman, R. Solids and surfaces: a chemist's view of bonding in extended structures; 1988.

(31) Fabini, D. H.; Stoumpos, C. C.; Laurita, G.; Kaltzoglou, A.; Kontos, A. G.; Falaras, P.; Kanatzidis, M. G.; Seshadri, R. Angew. Chemie - Int. Ed. 2016, 55 (49), 15392.

(32) Knutson, J. L.; Martin, J. D.; Carolina, N.; Uni, S.; Mitzi, D. B.; Watson, I. B. M. T. J.; Box, P. O.; Heights, Y.; York, N. 2005, 44 (13), 4699.

(33) Belisle, R. A.; Jain, P.; Prasanna, R.; Leijtens, T.; McGehee, M. D. ACS Energy Lett. 2016, 556.

(34) Glazer, A. M. Acta Crystallogr. Sect. B Struct. Crystallogr. Cryst. Chem. 1972, 28 (11), 3384.

(35) Whitfield, P. S.; Herron, N.; Guise, W. E.; Page, K.; Cheng, Y. Q.; Milas, I.; Crawford, M. K. Sci. Rep. 2016, 6 (October), 35685.

(36) Woodward, P. M. Acta Crystallogr. Sect. B Struct. Sci. 1997, 53 (1), 32.

(37) Meillaud, F.; Shah, A.; Droz, C.; Vallat-Sauvain, E.; Miazza, C. Sol. Energy Mater. Sol. Cells 2006, 90 (18-19), 2952.

(38) Zhao, B.; Abdi-Jalebi, M.; Tabachnyk, M.; Glass, H.; Kamboj, V. S.; Nie, W. A.; Pearson, J.; Puttisong, Y.; Godel, K. C.; Beere, H. E.; Ritchie, D. A.; Mohite, A. D.; Dutton, S. E.; Friend, R. H.; Sadhanala, A. Adv. Mater. 2016, 29 (2), 1604744.

(39) Manzoor, S.; Yu, Z. J.; Ali, A.; Ali, W.; Bush, K. A.; Palmstrom, A. F.; Bent, S. F.; McGehee, M. D.; Holman, Z. C. Sol. Energy Mater. Sol. Cells 2017, No. June, 1. 


\section{Table of Contents Graphic}

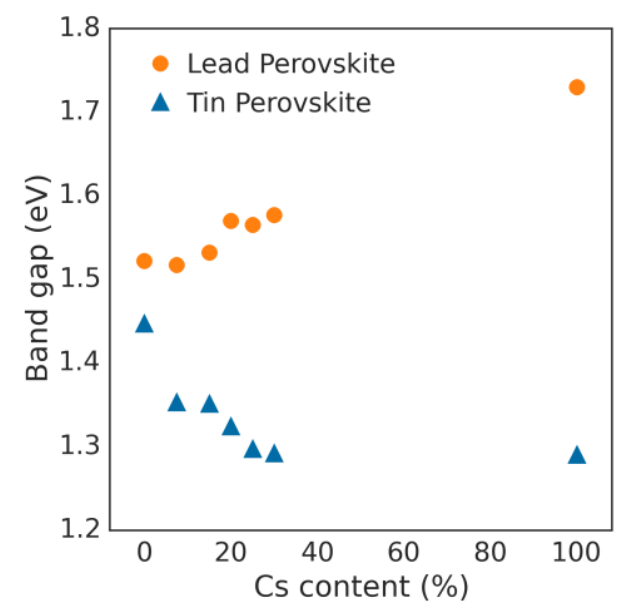

